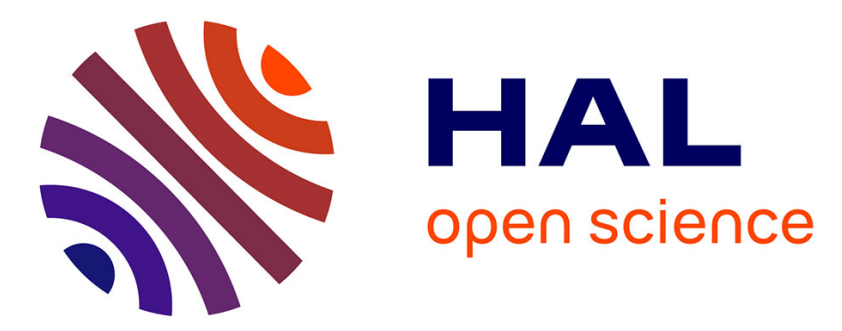

\title{
Spin-phonon coupling in the incommensurate magnetic ordered phase of orthorhombic TmMnO3
}

\author{
B.S. S Araújo, A.M. M Arévalo-López, C.C. C Santos, J.P. Attfield, C.W.A. \\ W A Paschoal, A.P. P Ayala
}

\section{- To cite this version:}

B.S. S Araújo, A.M. M Arévalo-López, C.C. C Santos, J.P. Attfield, C.W.A. W A Paschoal, et al.. Spin-phonon coupling in the incommensurate magnetic ordered phase of orthorhombic TmMnO3. Journal of Physics and Chemistry of Solids, 2021, 154, pp.110044. 10.1016/j.jpcs.2021.110044 . hal03436205

\section{HAL Id: hal-03436205 \\ https://hal.science/hal-03436205}

Submitted on 19 Nov 2021

HAL is a multi-disciplinary open access archive for the deposit and dissemination of scientific research documents, whether they are published or not. The documents may come from teaching and research institutions in France or abroad, or from public or private research centers.
L'archive ouverte pluridisciplinaire HAL, est destinée au dépôt et à la diffusion de documents scientifiques de niveau recherche, publiés ou non, émanant des établissements d'enseignement et de recherche français ou étrangers, des laboratoires publics ou privés. 


\title{
Spin-phonon coupling in the incommensurate magnetic ordered phase of orthorhombic $\mathrm{TmMnO}_{3}$
}

\author{
B.S. Araújo ${ }^{a, b}$, A.M. Arévalo-López ${ }^{b, c}$, C.C. Santos ${ }^{d}$, J.P. Attfield ${ }^{b}$, C.W.A. Paschoal ${ }^{a}$, A. \\ P. Ayala ${ }^{a, *}$ \\ a Physics Department, Universidade Federal do Ceará, PO Box 6030, Campus do Pici, 60455-760, Fortaleza, CE, Brazil \\ ${ }^{b}$ Centre for Science at Extreme Conditions (CSEC) and School of Chemistry, The University of Edinburgh, Peter Guthrie Tait Road, Edinburgh, EH9 3FD, United Kingdom \\ ' University of Lille, CNRS, Centrale Lille, University Artois, UMR 8181 - UCCS - Unité de Catalyse et Chimie du Solide, F-59000, Lille, France \\ d Physics Department, CCET, Universidade Federal do Maranhão, PO Box 65085-580, São Luís, MA, Brazil
}

\section{A RTICLE IN F O}

\section{Keywords:}

Spin-phonon coupling

High-pressure/high-temperature synthesis

Multiferroics

Raman spectroscopy

\begin{abstract}
A B S T R A C T
The search for materials with strong magnetoelectric coupling has been intense since interesting properties were discovered in the perovskite $\mathrm{TbMnO}_{3}$. Among the manganese-based perovskite family ( $\mathrm{RMnO}_{3}$ ), magnetic and electric orders coexist in the orthorhombic phase of the distorted perovskite $\mathrm{TmMnO}_{3}$. The antiferromagnetic phase induces ferroelectricity, leading to type-II multiferroic behavior. Although the temperature-dependent behavior of the Raman-active phonons of other members of the orthorhombic $\mathrm{RMnO}_{3}$ family was already reported, little information about orthorhombic $\mathrm{TmMnO}_{3}$ has been published. In this paper, bulk samples of orthorhombic $\mathrm{TmMnO}_{3}$ were obtained through HPHT treatment. Structural and magnetic properties of the synthesized samples were analyzed for phase confirmation. Temperature-dependent Raman spectra of bulk orthorhombic $\mathrm{TmMnO}_{3}$ revealed weak spin-phonon coupling in the incommensurate antiferromagnetic ordered phase around $44 \mathrm{~K}$. Raman spectra are also sensitive to the magnetic transition from the incommensurate to $\mathrm{E}$ type antiferromagnetic phase, where the frequency renormalization due to the spin-phonon coupling vanishes. This feature shows the existence of an orbital-spin-phonon coupling based on the magnetic ordering of the lowtemperature phase.
\end{abstract}

1. Introduction

Orthorhombic manganite perovskites $\mathrm{RMnO}_{3}$ (o-RMO) have been extensively investigated because of their exciting properties such as charge ordering, magnetoresistance, and multiferroicity [1-6]. At ambient conditions, large $\mathrm{R}^{3+}$ ionic radii $\left(r_{\mathrm{R}}\right)$ lead the $\mathrm{RMnO}_{3}$ perovskites to assume an orthorhombic structure, presenting an A-type antiferromagnetic (AFM) ordered phase $[4,7]$, whereas, in the case of smaller $r_{R}$, an hexagonal structure is preferred [8-10] and their magnetic order is an E-type AFM or incommensurate structure $[4,7]$. However, in compounds with small $r_{R}$, the orthorhombic phase can also be stabilized by simultaneous High-Pressure and High-Temperature (HP/HT) treatments [11].

Belonging to the class of small $r_{R}, \mathrm{TmMnO}_{3}$ (TMO) crystallizes in a hexagonal structure with a $\mathrm{P}_{3} \mathrm{~cm}$ space group. This structure can be described as layers of $\mathrm{MnO}_{5}$ bipyramids separated by $\mathrm{Tm}^{3+}$ layers [11]. Wang et al. [12] studied the ferroelectric behavior of hexagonal
$\mathrm{TmMnO}_{3}$ (h-TMO), reporting a ferro-paraelectric phase transition around $621 \mathrm{~K}$, whereas, Yen et al. [13] observed a magnetic transition around $84 \mathrm{~K}$. Later, Massa et al. [14] confirmed the electric and magnetic transitions showing a ferroelectric-magnetic coupling in the magnetically ordered phase using far-infrared emissivity and reflectivity measurements.

The orthorhombic phase of $\mathrm{TmMnO}_{3}$ (o-TMO), which is stabilized through HP/HT treatment, belongs to the Pbnm space group. This structure can be described as a distorted perovskite with corner-sharing $\mathrm{MnO}_{6}$ octahedra in a three-dimensional network with $\mathrm{Tm}^{3+}$ ions located at the free spaces between these octahedra. Using specific heat measurements, Tachibana et al. [15] reported a magnetic transition in o-TMO around $43 \mathrm{~K}$. Later, Pomjakushin $[16,17]$ classified the magnetic structure of O-TMO as incommensurate below $43 \mathrm{~K}$, observing a second magnetic transition at $32 \mathrm{~K}$ where the incommensurate structure becomes an E-type AFM. In the same study, the authors reported dielectric susceptibilities and pyroelectric measurements showing the existence of

\footnotetext{
* Corresponding author.

E-mail address: ayala@fisica.ufc.br (A.P. Ayala).
} 
a spontaneous electric polarization appearing below $32 \mathrm{~K}$, originated by lattice distortions due to the E-type AFM phase. These features evidence a multiferroic phase in o-TMO for bulk samples. Such multiferroic behavior was also confirmed by Salama et al. [10] using Mössbauer spectroscopy, while Han et al. [18] observed this phase in thin films. Moreover, these observations were confirmed through resonant X-ray analysis in bulk samples by Garganourakis et al. [19], and in thin films by Windsor et al. [20].

Recently, Shimamoto et al. [21] reported the room temperature Raman spectra of the orthorhombic $\mathrm{RMnO}_{3}(\mathrm{R}=\mathrm{Gd}-\mathrm{Lu})$ family (o-RMO) obtained as thin films on a $\mathrm{YAIO}_{3}$ substrate. This study shows the influence of chemical pressure along the b-axis since the $\mathrm{R}^{3+}$ ion change induces a shift of oxygen atoms on the ac plane, which increases the wavenumber of some vibrational modes compared to the values reported for bulk samples. Even though the Raman spectrum of o-TMO on a thin film was presented, neither information about the vibrational spectrum of bulk o-TMO nor its temperature dependence was reported.

The spin-phonon coupling in the o-RMO family was reported by Granado et al. [22] and Laverdi ère et al. [23], where the latter authors affirmed the coupling effect in small $r_{R}$ compounds and that the incommensurate AFM is negligible, whereas in E-type AFM ordered compounds, the coupling effect should be very weak or absent [23].

In TMO, the spin-phonon coupling was confirmed in the hexagonal phase using far-infrared spectroscopy [24]. In this study, the authors observed a phonon renormalization occurring at the magnetically ordered phase, where the renormalization effects were estimated to be around $5 \mathrm{~cm}^{-1}$.

The coupling between electric and magnetic ordering observed in hTMO suggests that the magnetic ordering can also couple with the lattice phonons in the case of o-TMO. Due to its sensitivity in detecting magnetic orderings through the coupling with the phonon lattice, mainly in perovskites compounds [25-27], Raman spectroscopy was employed to investigate the multiferroic properties of o-TMO by monitoring the spin-phonon coupling effect through its low-temperature magnetic transitions.

\section{Experimental session}

Polycrystalline o-TMO samples were obtained through an HP/HT treatment of h-TMO. The h-TMO precursor was synthesized by a solidstate reaction. Stoichiometric amounts of $\mathrm{Tm}_{2} \mathrm{O}_{3}$ (REacton, 99.99\%) and $\mathrm{Mn}_{2} \mathrm{O}_{3}$ (Aldrich, 99.99\%) were ground together in an agate mortar, and the mixture was calcined in a conventional oven at $1200{ }^{\otimes} \mathrm{C}$ for $24 \mathrm{~h}$ Subsequently, h-TMO pellets were ground and sealed into platinum capsules and treated at $1573 \mathrm{~K}$ under $6 \mathrm{GPa}$ for $60 \mathrm{~min}$ in a Walker-type multianvil press. After the reaction, the system was quenched to room temperature (RT), and the pressure was slowly released until atmospheric pressure. The resultant dark-green dense pellets were characterized by X-ray diffraction (XRD) on a BRUKER D8 diffractometer (Cu$\mathrm{Ka}_{1}$ radiation $1.5406 \AA$ ) to confirm the stabilization of the orthorhombic phase.

Magnetic measurements were carried out with a Quantum Design MPMS XL SQUID magnetometer. Magnetic susceptibility was recorded in zero-field cooled (ZFC) and field-cooled (FC) conditions, between 2 and $300 \mathrm{~K}$, with an applied magnetic field of 1000 Oe. Hysteresis loops were also measured at $2 \mathrm{~K}$.

Raman spectroscopy measurements were performed in a Jobin-Yvon T64000 Triple Spectrometer configured in a backscattered geometry and equipped with a liquid $\mathrm{N}_{2}$-cooled CCD detector. The $633 \mathrm{~nm}$ line of a HeNe gas laser operating at $20 \mathrm{~mW}$, focused on the sample by a longworking distance objective $(20 \times, 20.5 \mathrm{~mm})$, was used to excite the signal. The spectrometer slits were configured to obtain a spectra resolution lower than $1 \mathrm{~cm}^{-1}$. Temperature-dependent Raman measurements from 15 up to $300 \mathrm{~K}$ were carried out using a closed-cycle $\mathrm{He}$ cryostat in which a Lakeshore temperature controller model $330 \mathrm{~S}$ controlled the temperature with a precision of $0.1 \mathrm{~K}$. The Raman spectra were deconvoluted with Lorentzian functions using the Fityk software [28].

\section{Results}

The as-synthesized $\mathrm{TmMnO}_{3}$, obtained by solid-state reaction, pre-

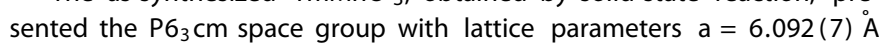
and $c=11.390(8) \stackrel{\circ}{A}$. Through an HP/HT treatment, the crystalline structure of the hexagonal TMO phase was converted into orthorhombic (Pbnm). The X-ray Rietveld refinement result for the orthorhombic phase presented the lattice parameters $a=5.231(8) \quad \AA, b=5.814(2) \quad \AA$, and $c=$ 7.323(5) $\AA$ (Fig. 1), which are in good agreement with the literature [11]. This phase is identified as an orthorhombically distorted-perovskite structure where the $\mathrm{Mn}^{3+}$ ions are octahedrally coordinated with six oxygen ions forming a distorted corner-sharing $\mathrm{MnO}_{6}$ network, where $\mathrm{Tm}^{3+}$ ions are located at the free spaces between these octahedra (inset of Fig. 1).

The temperature-dependent magnetic susceptibility of o-TMO samples is shown in figure S1(a). The overall signature of magnetic susceptibility obtained here is in good accordance with the one reported in the literature $[10,11,17]$. The magnetization signal as a function of the magnetic field measured at $2 \mathrm{~K}$ is shown in figure $\mathrm{S} 1(\mathrm{~b})$. This result shows a very sharp hysteresis loop with two very slight saturated magnetization points, and two states of spontaneous magnetization (inset of figure S1(b)). The same profile was reported by Yoshii et al. for h-TMO [29]. Despite the magnetic ordering transition in the Mn sublattice becoming more evident in specific heat analyzes for both hexagonal and orthorhombic phases $[10,11,17,29-31]$, the inverse of magnetic susceptibility (figure S1(c)) presents a deviation from linear paramagnetic behavior around $44 \mathrm{~K}$, whereas the first derivate of magnetic susceptibility (figure $\mathrm{S} 1(\mathrm{~d})$ ) presents a sudden decrease. The overall character of temperature-dependent magnetic features obtained here corroborates the antiferromagnetically ordered behavior of o-TMO reported in the literature.

The room temperature Raman spectrum of o-TMO is shown in Fig. 2. Based on group theory analysis, the distribution of Raman-active irre ducible representations of the $D_{2 h}$ factor group at the $\Gamma$ point of the Brillouin zone is $7 \mathrm{~A}_{\mathrm{g}} \otimes 5 \mathrm{~B}_{1 \mathrm{~g}} \otimes 7 \mathrm{~B}_{2 \mathrm{~g}} \otimes 5 \mathrm{~B}_{3 \mathrm{~g}}$ [32]. According to this analysis, 24 modes of mixed symmetries are predicted in the Raman spectra of the o-TMO polycrystalline sample. In our Raman analysis, we were able to identify 13 vibrational modes, the most intense one being located around $620 \mathrm{~cm}^{-1}$.

The assignments of the observed Raman modes can be made based on other o-RMO compounds [8,33-35]. In such studies, the authors have shown that the $\mathrm{MnO}_{6}$ phonon positions depend on the $\mathrm{R}^{3+}$ ionic radius $\left(r_{R}\right)$. Iliev et al. [34] reported a theoretical dynamic calculation model for the $r_{R}$ dependence of the phonon energy for the most intense $A_{g}(3)$, $A_{g}(1)$ and $B_{2 g}(1)$ bands, showing that the decrease in $r_{R}$ implies that the $A_{g}(3)$ frequency should be higher than the one of $A_{g}(1)$, while the $B_{2 g}(1)$ mode should be the most intense one.

In the case of o-TMO, the external vibrational modes are located below $200 \mathrm{~cm}^{-1}$, while those modes with higher wavenumbers are related to $\mathrm{MnO}_{6}$ octahedra internal vibrations (Table 1). The three very weak modes observed at 290,329 , and $343 \mathrm{~cm}^{-1}$ have $A_{g}(7), A_{g}(2)$, and $B_{2 g}(7)$ symmetries, respectively. The $A_{g}(7)$ and $B_{2 g}(7)$ modes are related to the basal octahedron oxygen motions along the $x$ and $z$ directions, while the $A_{g}(2)$ mode can be related to in-phase $\mathrm{MnO}_{6}$ rotations around the $y$-axis. The modes located around 404 and $493 \mathrm{~cm}^{-1}$ present symmetries $A_{g}(4)$ and $B_{2 g}(3)$ assigned as out-of-phase rotations along the $x$ axis and bending of $\mathrm{MnO}_{6}$, respectively. At higher wavenumbers, the mode located at $502 \mathrm{~cm}^{-1}$ originates from oxygen stretching having $A_{g}(1)$ symmetry, while the one located at $526 \mathrm{~cm}^{-1}$ has $A_{g}(3)$ symmetry, being related to $\mathrm{MnO}_{6}$ octahedra bending. Finally, the modes at 546 and $619 \mathrm{~cm}^{-1}$ have $\mathrm{B}_{2 \mathrm{~g}}$ symmetry and are assigned to in-phase $\mathrm{O}_{2}$ "scissorslike" and stretching motions, respectively. 


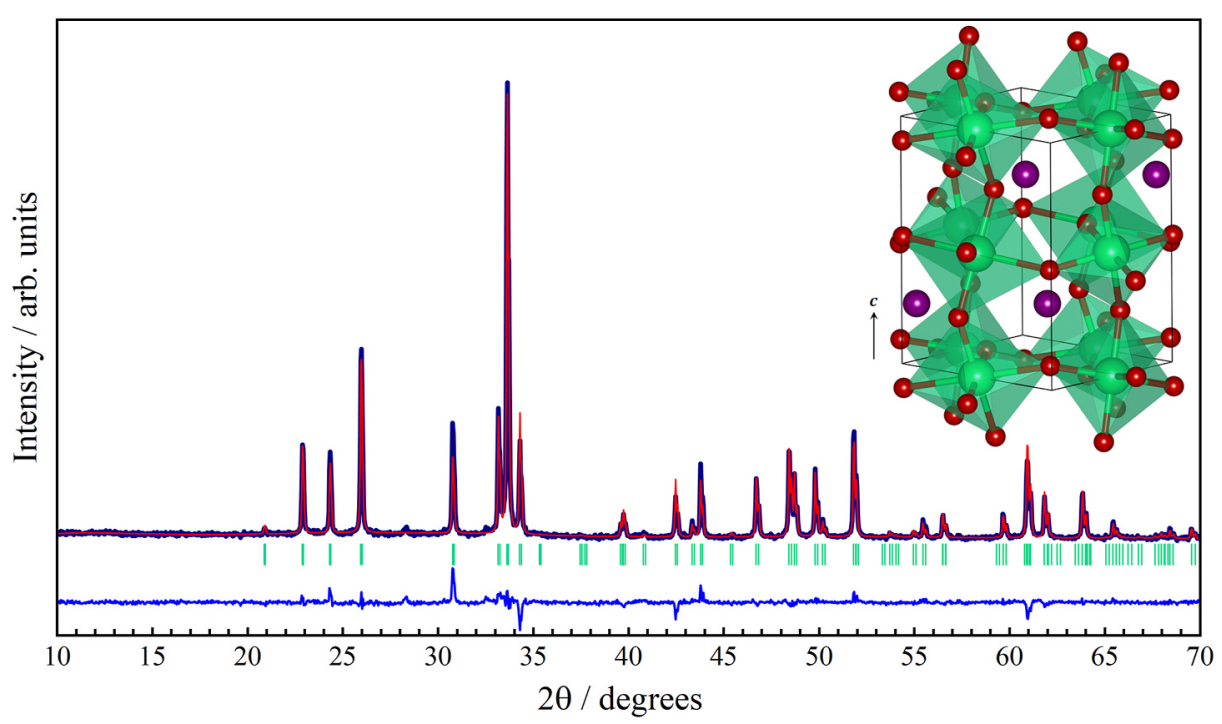

Fig. 1. Powder XRD pattern and Rietveld refinement of the o-TMO sample. The green bars indicate the reflection positions of o-TMO [11] (ICSD card number 162209).

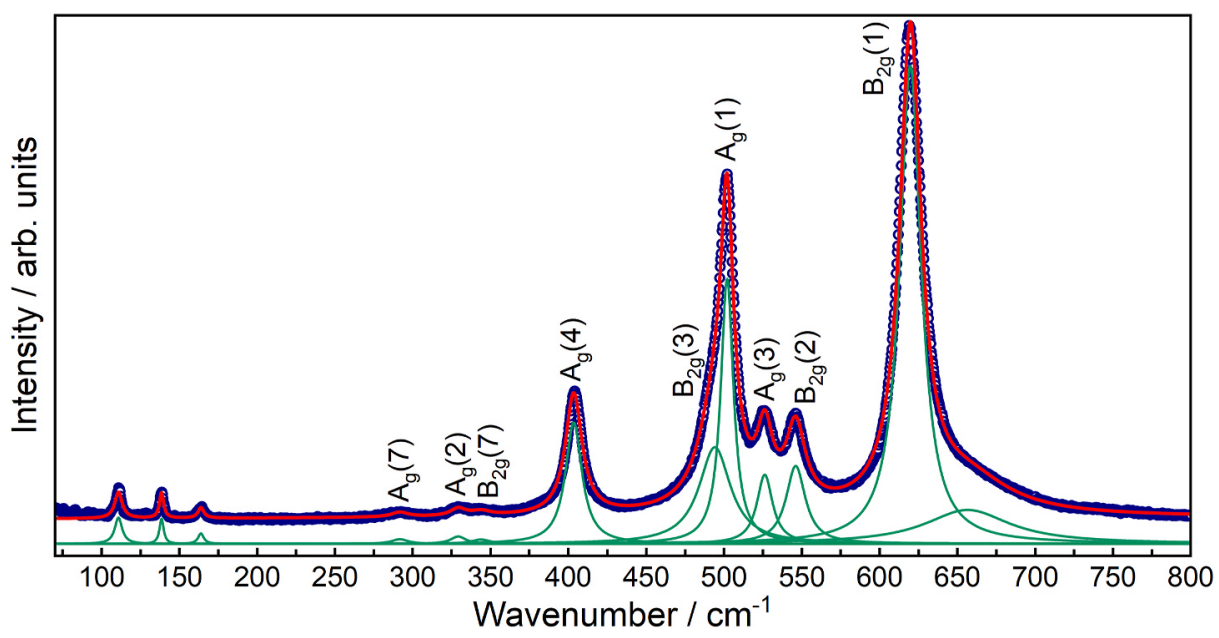

Fig. 2. Orthorhombic $\mathrm{TmMnO}_{3}$ Raman spectrum at room temperature.

Table 1

Assignment of active Raman modes observed for o-TmMnO .

\begin{tabular}{lll}
\hline Frequency $\left(\mathrm{cm}^{-1}\right)$ & Assignment & Motion \\
\hline 290 & $\mathrm{~A}_{\mathrm{g}}(7)$ & $\mathrm{O}_{1}(\mathrm{x})$ \\
329 & $\mathrm{~A}_{\mathrm{g}}(2)$ & In-phase $\mathrm{MnO}_{6} \mathrm{y}$ rotations \\
343 & $\mathrm{~B}_{2 \mathrm{~g}}(7)$ & $\mathrm{O}_{1}(\mathrm{z})$ \\
404 & $\mathrm{~A}_{\mathrm{g}}(4)$ & Out-of-phase $\mathrm{MnO}_{6} \times$ rotations \\
493 & $\mathrm{~B}_{2 \mathrm{~g}}(3)$ & Out-of-phase $\mathrm{MnO}_{6}$ bending \\
502 & $\mathrm{~A}_{\mathrm{g}}(1)$ & $\mathrm{O}_{2}$ antistretching \\
526 & $\mathrm{~A}_{\mathrm{g}}(3)$ & $\mathrm{MnO}_{6}$ bending \\
546 & $\mathrm{~B}_{2 g}(2)$ & In-phase $\mathrm{O}_{2}$ "scissors-like " \\
619 & $\mathrm{~B}_{2 g}(1)$ & In-plane $\mathrm{O}_{2}$ stretching \\
\hline
\end{tabular}

The only Raman investigation on o-TMO was recently reported by Shimamoto et al. [21] where they analyzed the Raman spectra of o-TMO thin films. In this study, the authors were only able to investigate the $A_{g}$ modes, and their respective positions are very close to those observed here. As stated, the subtle divergences in the phonon energies are due to the epitaxial film strain which shifts the Raman modes to higher wavenumbers when compared to bulk samples.

The o-TMO temperature-dependent Raman measurements were carried out in the temperature range between 300 and $15 \mathrm{~K}$. Because of resolution limitations, the temperature dependence of three $A_{g}$ and three $B_{g}$ modes with mixed symmetries (Fig. 3) were analyzed, while the modes observed between 280 and $360 \mathrm{~cm}^{-1}$ were too weak to be monitored. During the cooling process, the Raman spectra did not present any remarkable change in terms of additional or suppressed bands. Only the usual high-frequency shift and the sharpening of vibrational modes were observed. Therefore, the overall signature of Raman spectra is maintained within the investigated temperature range, implying that the orthorhombic phase of $\mathrm{TmMnO}_{3}$ did not undergo a structural phase transition at low temperatures.

In the absence of significant structural changes, the temperaturedependent behavior of a phonon of frequency $\omega$ is mainly governed by the phonon anharmonicity hardening. This behavior was described by Balkanski et al. [36], taking into account up to three phonon interactions. The temperature dependence of the wavenumber $(\omega(T))$ and full width at half maximum $(\Gamma(T))$ are given by equations (1) and (2), respectively.

$\omega(T)=\omega_{0}+A\left[1+\frac{2}{\left(e^{x}-1\right)}\right]+B\left[1+\frac{3}{\left(e^{y}-1\right)}+\frac{3}{\left(e^{y}-1\right)^{2}}\right]$ 


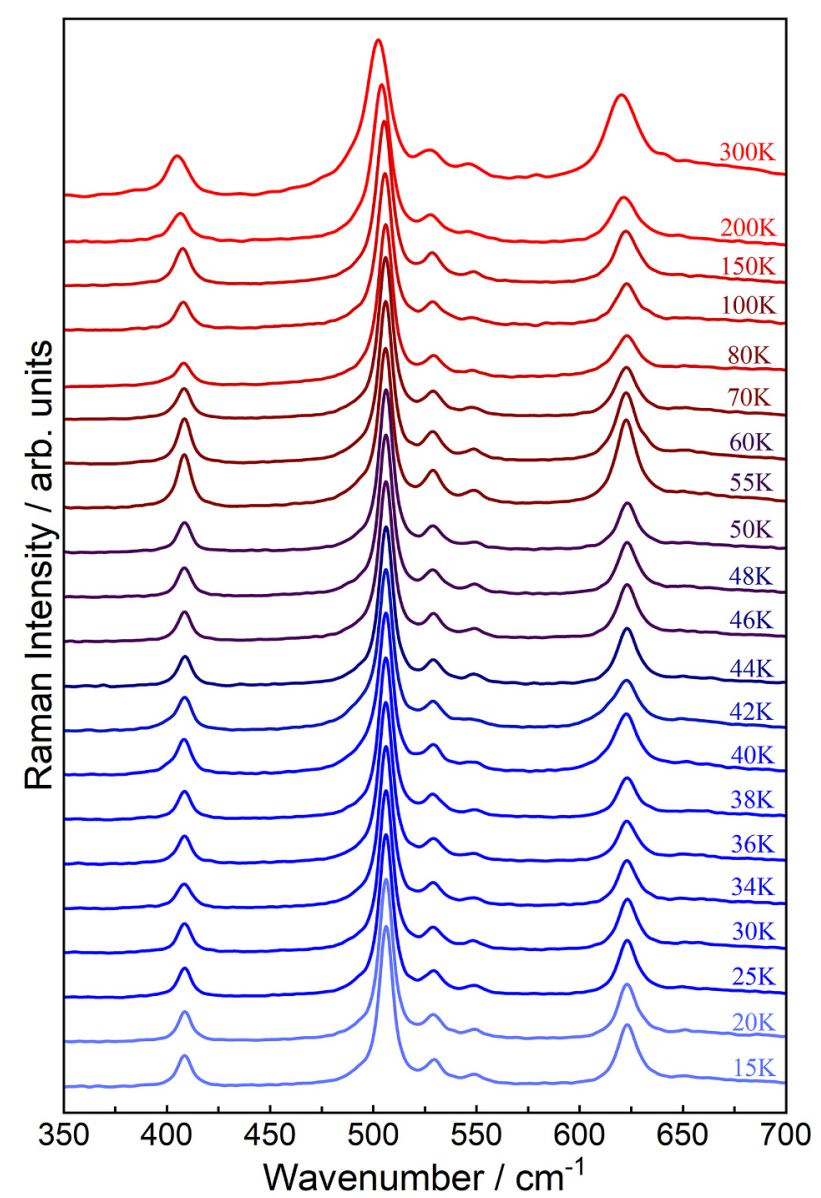

Fig. 3. Temperature dependence of the Raman spectra of o-TMO.

$\Gamma(T)=C\left[1+\frac{2}{\left(e^{x}-1\right)}\right]+D\left[1+\frac{3}{\left(e^{y}-1\right)}+\frac{3}{\left(e^{y}-1\right)^{2}}\right]$

where $\omega_{0}, A, B, C$, and $D$ are fitting parameters. The terms $x$ and $y$ are $\nabla \omega_{0} / 2 K_{B} T$ and $\nabla \omega_{0} / 3 K_{B} T$, respectively.

Fig. 4 shows the temperature dependence of selected phonon wavenumbers and full width at half maximum (FWHM) compared to Balkanski 's model. In contrast to other phonons monitored in this study (see figure S2), these phonons closely follow the anharmonic model down to $44 \mathrm{~K}$, where clear departures from the theoretical curve are observed. At this temperature, a magnetic transition has been reported from a paramagnetic to an incommensurate AFM phase. Thus, the sudden frequency hardening of these vibrational modes, starting around the temperature at which the spin moments become aligned, is a clear indication of a spin-phonon coupling effect in o-TMO.

It is important to note that Pomjakushin and co-workers [17] reported that o-TMO experiences a coupling effect between the lattice parameters and the magnetic ordering below $T_{N}$, showing the existence of a lattice distortion stronger than those observed in other members of the o-RMO family. Based on this feature, the renormalization effects on phonon positions observed here could also be induced by the lattice distortions instead of a spin-phonon coupling. Once FWHM is related to the phonon lifetime [37], the effects directly connected to lattice variations do not influence this parameter, allowing us to distinguish phenomena generated by the spin-lattice coupling from those originating in other kinds of coupling effects, such as the spin-phonon. Thus, the study of the phonon FWHM becomes crucial to solve the ambiguity related to the origin of the frequency renormalization. The temperature dependence of the FWHM of selected phonons is also presented in Fig. 4, where departures from the anharmonic model can also be noticed
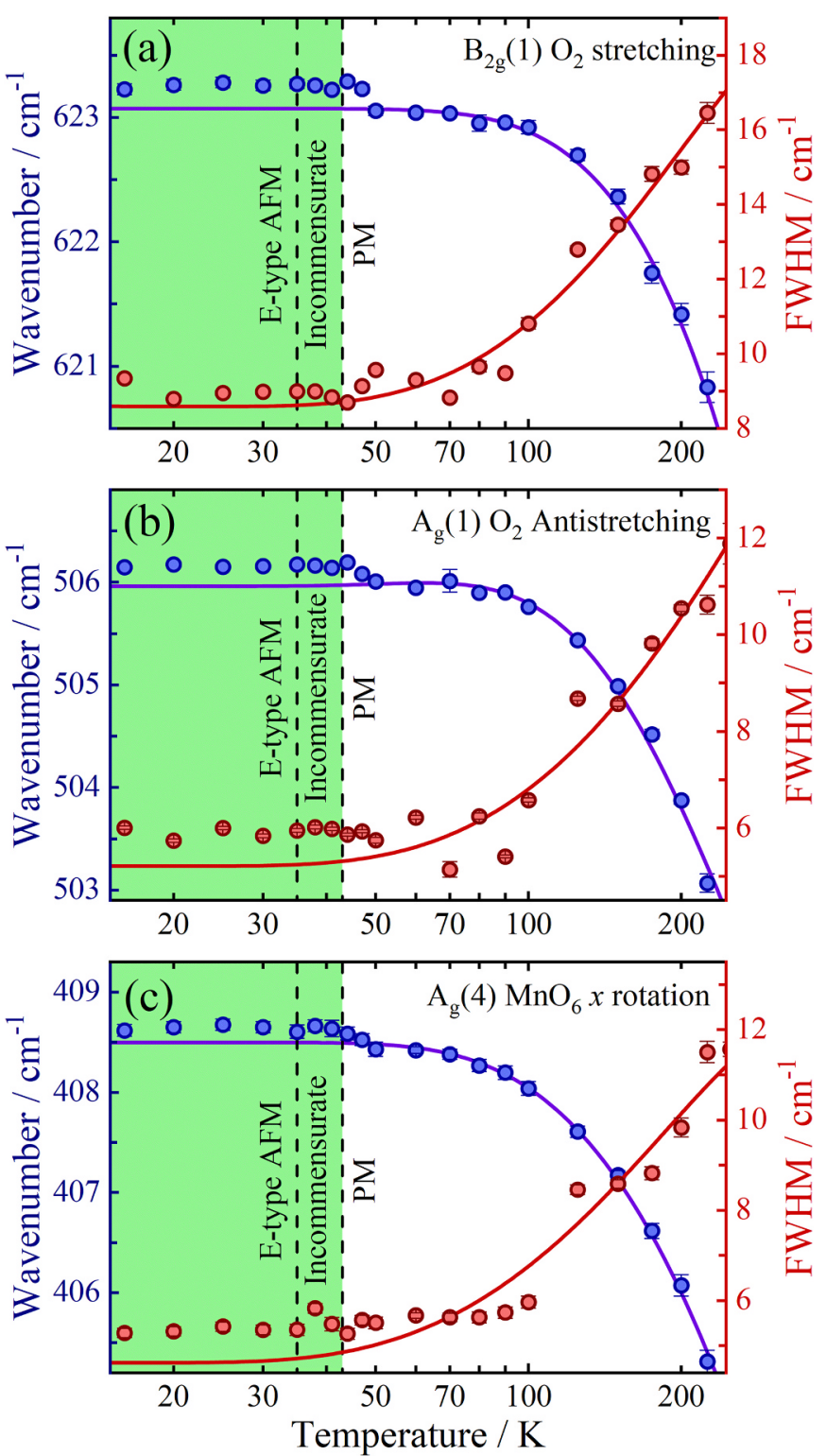

Fig. 4. Temperature dependence of selected phonon wavenumber and FWHM of orthorhombic $\mathrm{TmMnO}_{3}$. Solid purple and red lines indicate the fit based on Balkanski 's models for phonon positions and FWHM, respectively. The dashed lines locate the magnetic transitions reported in the literature.

around $45 \mathrm{~K}$, suggesting that spin-phonon coupling originates the observed effects.

Additional evidence in favor of the spin-phonon origin of the phonon renormalization can be given by considering lattice distortions. The phonon frequency shift induced by changes in ionic binding energies due to lattice distortions can be approximated based on the Grüneisen law: $\delta \omega_{a}(T)=-\gamma_{a} \omega_{0}\left(\Delta V / V_{0}\right)$, where $\gamma_{a}$ is the Grüneisen parameter for the normal mode $a$. Thus, if the phonon energy change is due to lattice distortions, it could be expected to be correlated with the directions or planes (A) closely related to the atomic displacements of a given phonon: $\delta \omega_{a}(T)=-\gamma_{a} \omega_{0}\left(\Delta A / A_{0}\right)$ [38].

Fig. 5 shows the frequency change relative to the lower measured temperature $(\delta \omega(T)=\omega(16 K)-\omega(T))$ for the $A_{g}(4), A_{g}(1)$ and $B_{2 g}(1)$ modes of o-TMO (blue circles) compared to the variation of $A=b \times c$ (Fig. $5(\mathrm{a})$ ) and $A=\mathrm{a} \times \mathrm{c}$ planes (Fig. 5 (b) and (c)), calculated from the data reported by Pomjakushin et al. [17]. As can be seen, there is no correlation between all three coupled phonons with their respective expansion planes in the temperature range of the lattice distortions 

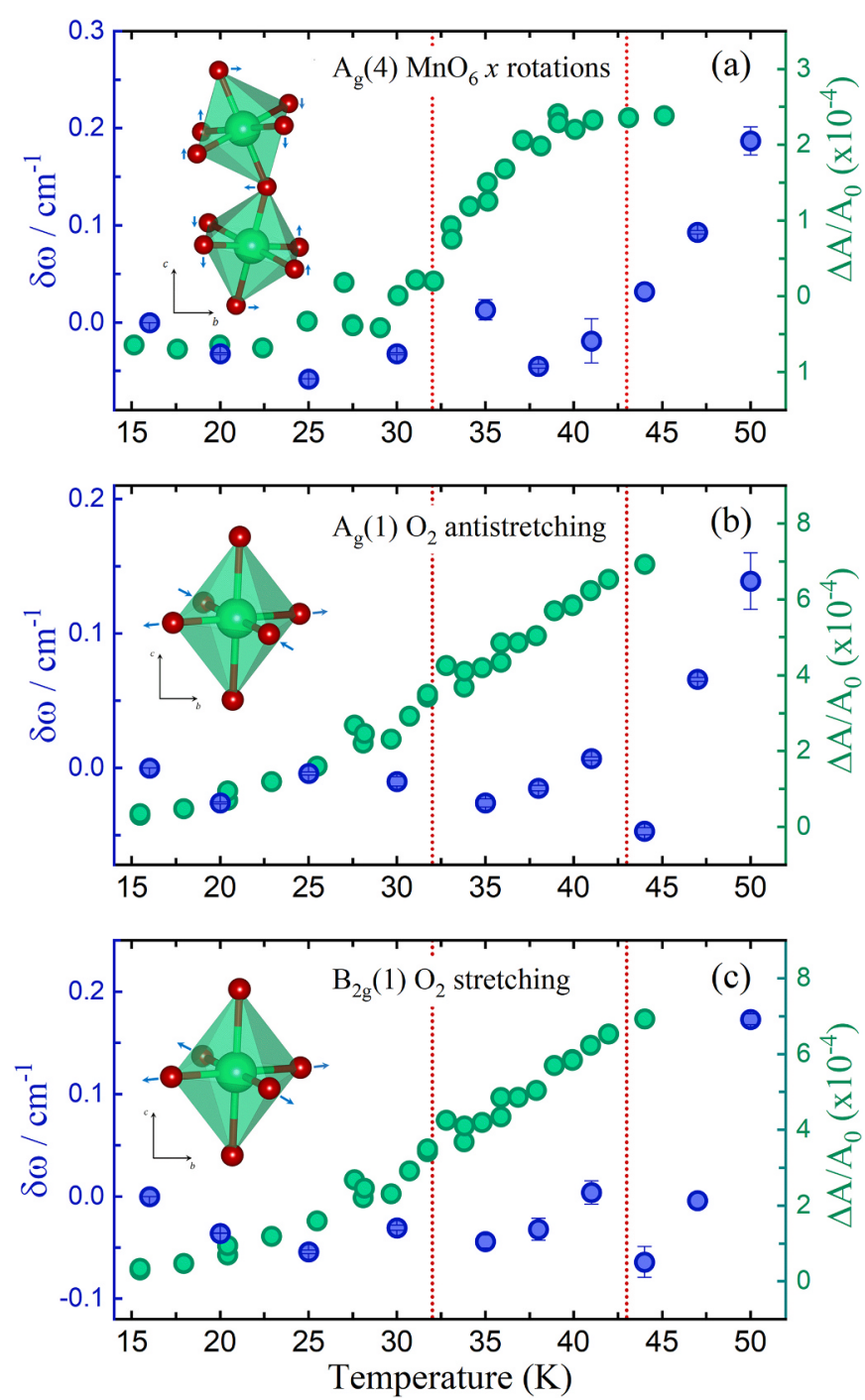

Fig. 5. Comparison of the temperature-dependence of the phonon energy $(\delta \omega(T)=\omega(16 K)-\omega(T))$ and the lattice planes corresponding to (a) $A_{g}(4)$ $(A=b \times c)$, (b) $A_{g}(1)$ and $(c) B_{2 g}(1)(A=a \times b)$. The lattice parameters were obtained from Ref. [17].

induced by the magneto-structural coupling. These results also support the spin-phonon origin of the frequency renormalization.

Like o-TMO, other members of the o- $\mathrm{RMO}_{3}$ family also showed a spin-phonon coupling starting around the temperatures at which the magnetically ordered phase arises [23,38]. However, unlike other o-RMO ${ }_{3}$ compounds, the o-TMO phonons presented a hardening effect on phonon positions instead of a softening, as observed by Laverdi ère et al. [23]. Also, the magnitude of the phonon renormalization due to spin-phonon coupling in O-TMO is weaker in comparison to those observed for $\mathrm{PrMnO}_{3}$ [23] and $\mathrm{ErMnO}_{3}$ [39], but still comparable to the one of the $A_{g}(1)$ phonon of $\mathrm{DyMnO}_{3}$ [23]. In contrast to our observations, previous reports claim that the spin-phonon coupling is negligible in compounds with small $r_{R}$ and incommensurate AFM ordering. Conversely, the phonon wavenumber and FWHM o-TMO indicate a weak but not negligible spin-phonon coupling rising near the incommensurate AFM ordering temperature.

The renormalization effects experienced by the o-TMO phonons can be observed slightly above $T_{N}$. Laverdi ère et al. [23] highlighted this as a common effect also found in other compounds such as $\mathrm{LaMnO}_{3}$ [22], $\mathrm{PrMnO}_{3}, \mathrm{NdMnO}_{3}, \mathrm{SmMnO}_{3}$, and $\mathrm{DyMnO}_{3}$ [23], in which the spin-phonon coupling also starts well above (around $30 \mathrm{~K}$ ) the magnetic ordering temperature. In such cases, the authors suggest that the renormalization above $T_{N}$ could be explained by in-phase ferromagnetic interactions existing even in paramagnetic phases [23].

The renormalization effect of a phonon frequency $(\Delta \omega)$ due to spinphonon coupling can be modeled by taking into account the static spinspin correlation average function $[40,41]$ as proposed by Granado et al. [22], which leads to the following relation:

$\Delta \omega \nabla \omega-\omega_{0}=\frac{2}{m \omega} \sum_{i, j>i} \frac{\partial^{2} J_{i j}}{\partial u^{2}} S_{i} \Phi_{j}$

where $\omega$ is the renormalized phonon frequency due to the spin-phonon coupling, $\omega_{0}$ is the phonon frequency in the absence of a coupling effect, $\mathrm{m}$ is the oxygen mass, $\mathrm{u}$ is the atomic displacement during the vibrational motion, $\mathrm{J}_{\mathrm{ij}}$ is the exchange integral between the $\mathrm{i}^{\text {th }}$ and the $j^{\text {th }}$ magnetic ion, and $S_{i} \bigotimes_{j}$ denotes the statistical-mechanical average for adjacent spin moments.

This model has been employed to describe the spin-phonon coupling in several perovskites such as $\mathrm{Gd}\left(\mathrm{Co}_{1 / 2} \mathrm{Mn}_{1 / 2}\right) \mathrm{O}_{3}$ [42], $\mathrm{Y}_{2} \mathrm{NiMnO}_{6}$ [43], and $\mathrm{BiCrO}_{3}$ [44], as well as in non-perovskite compounds like $\mathrm{NiO}$ [45] and $\mathrm{Cu}_{2} \mathrm{OCl}_{2}$ [46]. However, in the case of incommensurate magnetic ordering, an extension of this model becomes necessary. Kimura et al. [7], considering the inclusion of the nearest-neighbor (NN) and the next-nearest-neighbor (NNN) interactions, have rewritten the phonon renormalization as:

$\Delta \omega=\frac{1}{2 m \omega} \sum_{r} \frac{\partial^{2} J(r)}{\partial u^{2}} S_{i} \Phi_{i+r}$

For an incommensurate ordering with propagation vector k, Laverdière et al. [23] proposed the following spin correlation function

$S_{i} \otimes S_{i+r}=K(T) \cos (2 \pi k \otimes r)$

where $K(T)$ is a temperature-dependent prefactor $\left[K(T) \otimes M_{\text {sublatt }}^{2}(T)\right]$.

Pomjakushin et al. [17] showed that the first magnetic transition of O-TMO drives the paramagnetic phase into an incommensurate AFM phase at $42 \mathrm{~K}$. The incommensurate phase has $k=\left(k_{x}, 0,0\right)$, with $k_{x}$ varying from 0.45 to 0.5 , when the incommensurate AFM structure becomes an E-type AFM structure. $\mathrm{Mn}^{3+}$ ions can be classified according to the o-TMO magnetic structure as follows, $N N$ : four ions $\left(J_{1}\right)$ placed at $r= \pm 0.5 x \pm 0.5 z$, and NNN: two ions $\left(J_{2}\right)$ at $r= \pm x$ and two ions $\left(J_{3}\right)$ at $r= \pm z$. Thus, the resulting phonon renormalization is given by Ref. [23] $\left(D_{\mathrm{i}}=\partial^{2} \mathrm{~J}_{\mathrm{i}} / \partial \mathrm{u}^{2}\right)$ :

$\Delta \omega=\frac{K(T)}{m \omega}\left[2 D_{1} \cos \left(\pi k_{x}\right)+D_{2} \cos \left(2 \pi k_{x}\right)+D_{3}\right]$

In the case of the E-type magnetic phase of o-TMO with $\mathrm{k}_{\mathrm{x}}=0.5$, the $\mathrm{NN}$ contributions cancel themselves since each $\mathrm{Mn}^{3+}$ ion is surrounded by two sets of ions with opposite spin moments [17,23], and equation (6) becomes:

$\Delta \omega=\frac{2}{m \omega}\left[D_{3}-D_{2}\right]\left(\frac{M(T)}{M_{0}}\right)^{2}$

Based on these considerations, in both magnetically ordered phases of o-TMO, the phonon renormalization should be proportional to $\left(M(T) / M_{0}\right)^{2}$. Thus, in Fig. 6 , the $\Delta \omega$ for the selected phonons $\left(A_{g}(4)\right.$, $A_{g}(1)$ and $\left.B_{2 g}(1)\right)$ is plotted as a function of $\left(M(T) / M_{0}\right)^{2}$. As was discussed previously, a non-zero $\Delta \omega$ is observed just below $T_{N}(\sim 50 K)$, which is characterized by the rising of the phonon renormalization without an apparent linear trend. In fact, a simple proportionality constant cannot be expected in this region since $\Delta \omega$ not only depends on $\left(M(T) / M_{0}\right)^{2}$, but also on the $k_{x}$ value of the incommensurate phase (eq. (6) ). A different linear dependence is also observed rising at $\sim 35 \mathrm{~K}$, where both trends meet. This temperature agrees quite well with the lock-in phase transition into the E-type AFM phase, where eq. (7) should 

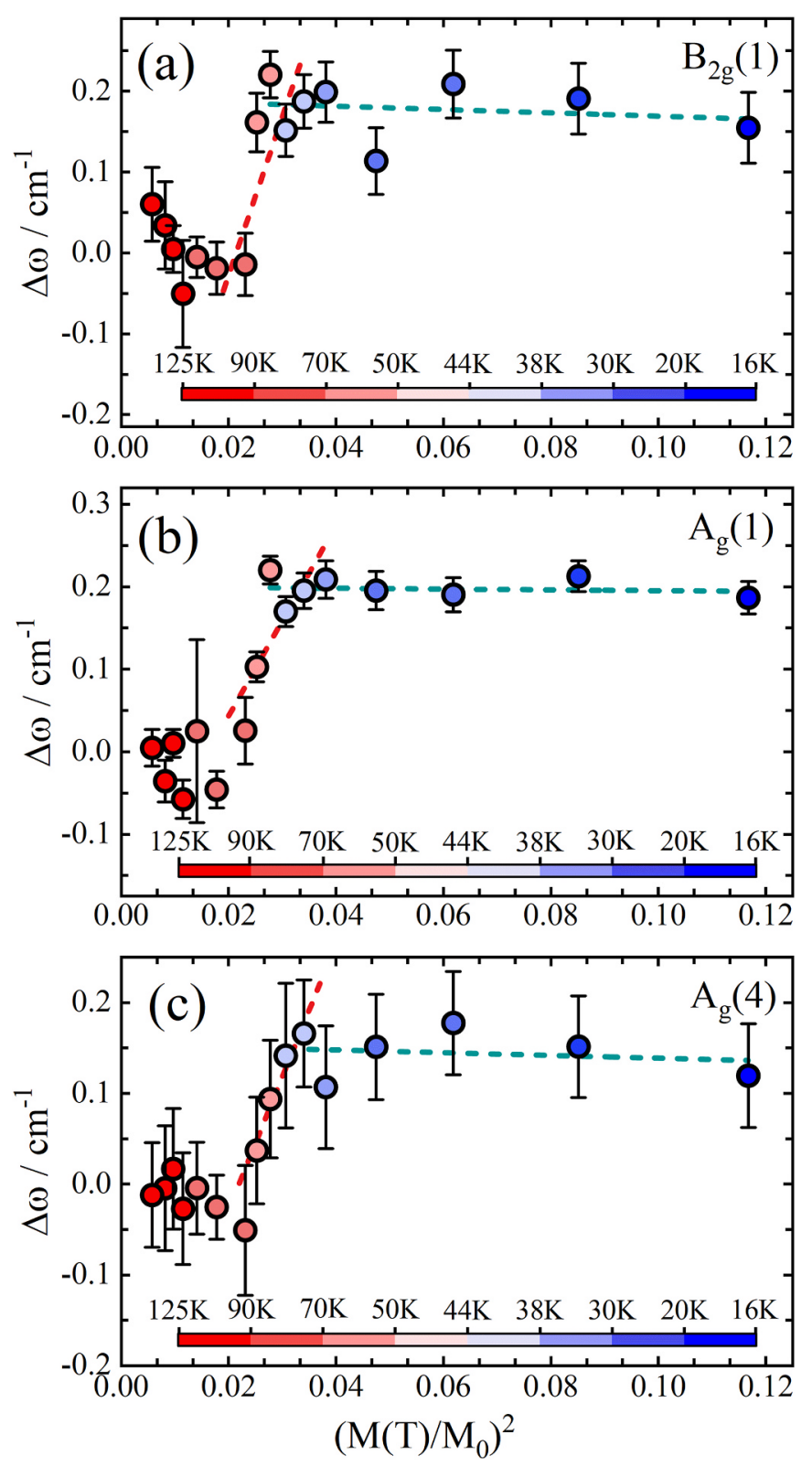

Fig. 6. Panels a-c: Temperature dependence of the departure from anharmonic behavior of selected phonons as a function of $\left(M(T) / M_{0}\right)^{2}$, the red and dark green dashed lines are, respectively, the linear fit of the renormalization in the IC and E-type AFM phases.

describe the $\Delta \omega$ behavior. By fitting the AFM region with a linear function, the slope obtained for $B_{2 g}(1), A_{g}(1)$ and $A_{g}(4)$ phonons were $0.2(5),-0.1(2)$, and $-0.2(4) \mathrm{cm}^{-1}$, respectively. These very small values suggest that $\Delta \omega$ as a function of $\left(M(T) / M_{0}\right)^{2}$ becomes approximately constant, implying that the factor $\left[D_{3}-D_{2}\right]$ in eq. (7) should be neglectable in the E-type AFM phase, in good agreement with the Laverdière et al. observations [23].

The neglectable spin-phonon coupling in the E-type AFM phase is evidenced in Fig. 4 since the phonon wavenumber follows Balkanski's model, but slightly shifted towards higher values. A similar phenomenon also can be observed in the $384 \mathrm{~cm}^{-1}$ mode of $\mathrm{TbMnO}_{3}$ and the 516.5 and $494 \mathrm{~cm}^{-1}$ modes of $\mathrm{DyMnO}_{3}$ [38]. In both cases, the renormaliza tion of phonon frequencies starts around the temperatures at which an incommensurate magnetic structure rises $[6,47,48]$. Also, after the second magnetic transition, the renormalization process becomes constant. In such cases, the phonon frequency renormalization saturation suggests that the spin-phonon coupling is not the only factor involved in the temperature dependence of the phonon wavenumbers.

Aiming to investigate such effects, $\mathrm{Xu}$ et al. [49] developed a theoretical model considering the possible contributions of the orbital ordering to the spin-phonon coupling. Considering this contribution, the frequency shift $\Delta \omega_{\text {OSP }}(T)$ can be rewritten as a function of the effective spin-phonon coupling $\left(J_{\mathrm{ij}}^{\mathrm{s}-\mathrm{ph}}\right)$ and the effective orbital-spin-phonon coupling $\left(J_{\mathrm{ij}}^{\circ \circ}\right)$ :

$\Delta \omega_{O S P}(T) \approx \frac{2}{m \omega} \sum_{i, j>i}\left(\frac{\partial^{2} J_{i j}^{s-p h}}{\partial u^{2}} S_{i} S_{j}+4 \frac{\partial^{2} J_{i j}^{o o}}{\partial u^{2}} S_{j} S_{j} \tau_{i} \tau_{j}\right)$

where $\tau_{i} \tau_{j}$ are the orbital correlation operators for the $i$ and $j \mathrm{Mn}^{3+}$ ions. By using a multivariate Taylor expansion $\sum_{i, j}\left(S_{i} S_{j} T_{i} T_{j}\right) \approx \sum_{i, j}\left(S_{i} S_{j} T_{i} T_{j}+\right.$ $S_{i} S_{j} \delta \tau+\tau_{i} \tau_{j} \delta S$ ), where $\delta \tau \otimes \tau_{i} \tau_{j}-\left.\tau_{i} \tau_{j}\right|_{S_{i} S_{j}=S_{i} S_{j}}$ and $\delta S \otimes S_{i} S_{j}-\left.S_{i} S\right|_{\tau_{i} T_{j}=\tau_{i} \tau_{j}}$ it is possible to establish an expression for the frequency shift due to the combined couplings [38,49]:

$\Delta \omega_{\text {OSP }}(T) \approx \frac{2}{m \omega} \sum_{i, j>i}\left[\left(\frac{\partial^{2} J_{i j}^{s-p h}}{\partial u^{2}}+4 \frac{\partial^{2} J_{i j}^{o o}}{\partial u^{2}}\left(\tau_{i} \tau_{j}+\delta S\right)\right) S_{i} S_{j}+4 \frac{\partial^{2} J_{i j}^{o o}}{\partial u^{2}} \tau_{i} \tau_{j} \delta S\right]$

This model was previously applied to describe the spin-phonon coupling of A-type AFM materials $[38,49]$. However, it needs to be modified in the case of the incommensurate to E-type magnetic transition based on eq. (6) [23]. Thus, for an incommensurate magnetic order, the $\Delta \omega(T)$ renormalization of coupled phonon modes can be rewritten as:

$$
\begin{aligned}
\Delta \omega_{\text {OSP }}(T) \approx & {\left[\left(2\left(D_{1}+D_{1}^{\circ \circ}\right) \cos \left(\pi k_{x}\right)+\left(D_{2}+D_{2}^{\circ \circ}\right) \cos \left(2 \pi k_{x}\right)\right.\right.} \\
& \left.\left.+\left(D_{3}+D_{3}^{\circ \circ}\right)\right) K(T)+D_{4}^{\mathbb{Q}}\right]
\end{aligned}
$$

where $D_{i}^{\circ o}=4\left(\tau_{i} \tau_{j}+\delta S\right) \partial^{2} J_{i j}^{\circ o} / \partial u^{2}$ and $D_{4}^{\bigotimes_{4}}=4 \delta S \sum \tau_{i} \tau_{j} \partial^{2} J_{i j}^{\circ o} / \partial u^{2}$. This result does not change the previous discussion about the spin-phonon coupling in the incommensurate phase because of the complex dependence on $\mathrm{k}_{\mathrm{x}}$. However, in the E-type AFM phase $\left(\mathrm{k}_{\mathrm{x}}=0.5\right)$, eq. (10) is reduced to:

$\Delta \omega_{\mathrm{OSP}}(T) \approx \frac{2}{m \omega}\left[\left(D_{3}-D_{2}+D_{3}^{\circ \circ}-D_{2}^{\circ \circ}\right)\left(\frac{M(T)}{M_{0}}\right)^{2}+4 D_{4}^{\boxplus}\right]$

The last equation has a linear dependence on the square of sublattice magnetization, where the intercept is related to the effective orbitalspin-phonon coupling ( $\left.\mathrm{k}_{\mathrm{OSP}}\right)$ and should be non-zero only in cases where this interaction exists. Previous reports have proposed that $D_{3} \otimes$ $D_{2}$ in the E-type AFM phase based on the observation of a neglectable spin-phonon coupling [23]. However, the observed null slope also requires the same condition for the contribution of the orbital-spin-phonon coupling $\left(D_{3}^{\circ \circ} \otimes D_{2}^{\circ \circ}\right)$. On the other hand, the three phonons shown in Fig. 6 have a finite intercept of $0.19(3), 0.20(1)$, and $0.16(3) \mathrm{cm}^{-1}$ for $B_{2 g}(1), A_{g}(1)$, and $A_{g}(4)$, respectively. These values are similar but smaller than those reported by Mansouri et al. [38], agreeing with the proposal that the $k_{\text {OSP }}$ value decreases with the ionic radius of the rare-earth in the o-RMO family.

\section{Conclusions}

The spin-phonon coupling in bulk o-TMO was explored by lowtemperature Raman spectroscopy measurements. Our results revealed that below $48 \mathrm{~K}$, the $A_{g}(4), A_{g}(3)$, and $B_{2 g}(1)$ phonons temperature dependence depart from the model based on the anharmonicity, exhibiting renormalization effects on the frequency and FWHM parameters. These features coincide with the paramagnetic to incommensurate AFM phase transition showing a well-defined spin-phonon coupling in contradiction to previous assumptions. The $\Delta \omega$ vs 
$\left(M(T) / M_{0}\right)^{2}$ dependence reveals a complex behavior in the incommensurate phase. However, approximately at the lock-in transition into the E-type magnetic structure, the frequency shift becomes constant. Based on previous studies, a model was proposed where the phonon renormalization has a contribution from an orbital-spin-phonon coupling, which becomes the dominant effect in the E-type AFM phase. The low magnitude of phonon frequency renormalization indicates the spinphonon coupling on o- $\mathrm{TmMnO}_{3}$ is weaker than those reported for its hexagonal phase, and other o-RMO compounds, but it is still comparable with those of the o-RMO family, such as $\mathrm{DyMnO}_{3}$.

\section{CRediT authorship contribution statement}

B.S. Araújo: Conceptualization, Methodology, Investigation, Formal analysis, Visualization, Writing - original draft, Writing - review \& editing. A.M. Arévalo-L ópez: Investigation, Methodology, Validation, Formal analysis, Writing - review \& editing. C.C. Santos: Methodology, Investigation, Resources. J.P. Attfield: Conceptualization, Resources, Writing - review \& editing. C.W.A. Paschoal: Conceptualization, Investigation, Validation, Supervision, Writing - review \& editing. A.P. Ayala: Conceptualization, Resources, Validation, Writing - review \& editing, Supervision.

\section{Declaration of competing interest}

The authors declare that they have no known competing financial interests or personal relationships that could have appeared to influence the work reported in this paper.

\section{Acknowledgments}

This study was partially financed by the Brazilian Agencies CAPES (Finance Code 001), FUNCAP (PRONEX PR2-0101-00006.01.00/15), and CNPq (Grant No. 427478/2016-2).

Appendix A. Supplementary data

Supplementary data to this article can be found online at

\section{References}

[1] N.A. Spaldin, R. Ramesh, Advances in magnetoelectric multiferroics, Nat. Mater. 18 (2019) 203-212, https://doi.org/10.1038/s41563-018-0275-2

[2] H.Y. Hwang, S.W. Cheong, P.G. Radaelli, M. Marezio, B. Batlogg, Lattice effects on the magnetoresistance in doped $\mathrm{LaMnO}_{3}$, Phys. Rev. Lett. 75 (1995) 914-917, https://doi.org/10.1103/PhysRevLett.75.914

[3] T. Kimura, T. Goto, H. Shintani, K. Ishizaka, T. Arima, Y. Tokura, Magnetic control of ferroelectric polarization, Nature 426 (2003) 55-58, https://doi.org/10.1038/ nature02018.

[4] T. Goto, T. Kimura, G. Lawes, A.P. Ramirez, Y. Tokura, Ferroelectricity and giant magnetocapacitance in perovskite rare-earth manganites, Phys. Rev. Lett. 92 (2004) 257201, https://doi.org/10.1103/PhysRevLett.92.257201

[5] E. Baldini, T. Kubacka, B.P.P. Mallett, C. Ma, S.M. Koohpayeh, Y. Zhu, C. Bernhard, S.L. Johnson, F. Carbone, Lattice-mediated magnetic order melting in $\mathrm{TbMnO}_{3}$ Phys. Rev. B 97 (2018) 125149, https://doi.org/10.1103/PhysRevB.97.125149

[6] M. Kenzelmann, A.B. Harris, S. Jonas, C. Broholm, J. Schefer, S.B. Kim, C.L. Zhang, S.-W. Cheong, O.P. Vajk, J.W. Lynn, Magnetic inversion symmetry breaking and ferroelectricity in $\mathrm{TbMnO}_{3}$, Phys. Rev. Lett. 95 (2005), 087206, https://doi.org/ 10.1103/PhysRevLett.95.087206

[7] T. Kimura, S. Ishihara, H. Shintani, T. Arima, K.T. Takahashi, K. Ishizaka, $\mathrm{Y}$. Tokura, Distorted perovskite with $\mathrm{e}_{\mathrm{g}}^{1}$ configuration as a frustrated spin system, Phys. Rev. B 68 (2003), https://doi.org/10.1103/PhysRevB.68.060403 , 060403.

[8] L. Martín-Carr ón, A. De Andrés, M.J. Martínez-Lope, M.T. Casais, J.A. Alonso, Raman phonons and light scattering in $\mathrm{RMnO}_{3}(\mathrm{R}=\mathrm{La}, \mathrm{Pr}, \mathrm{Nd}, \mathrm{Ho}, \mathrm{Er} \mathrm{Tb}$ and $\mathrm{Y})$ orthorhombic and hexagonal manganites, J. Alloys Compd. 323-324 (2001) 494-497, https://doi.org/10.1016/50925-8388(01)01047-7

[9] J.-S. Zhou, J.B. Goodenough, J.M. Gallardo-Amores, E. Morán, M.A. Alario-Franco, R. Caudillo, Hexagonal versus perovskite phase of manganite $(R=Y, H o, E r, T m$, Yb, Lu), Phys. Rev. B 74 (2006), https://doi.org/10.1103/PhysRevB.74.014422 014422 .

[10] H.A. Salama, G.A. Stewart, W.D. Hutchison, K. Nishimura, D.R. Scott, H.S. C. O'Neill, A 169Tm-M össbauer spectroscopy investigation of orthorhombic phase
o-TmMnO 3 , Solid State Commun. 150 (2010) 289-291, https://doi.org/10.1016/j. Ssc.2009.11.002

[11] K. Uusi-Esko, J. Malm, N. Imamura, H. Yamauchi, M. Karppinen, Characterization of $\mathrm{RMnO}_{3}(\mathrm{R}=\mathrm{Sc}, \mathrm{Y}, \mathrm{Dy}$-Lu): high-pressure synthesized metastable perovskites and their hexagonal precursor phases, Mater. Chem. Phys. 112 (2008) 1029-1034, https://doi.org/10.1016/j.matchemphys.2008.07.009

[12] L.J. Wang, S.M. Feng, J.L. Zhu, R.C. Yu, C.Q. Jin, W. Yu, X.H. Wang, L.T. Li, Ferroelectricity of multiferroic hexagonal $\mathrm{TmMnO}_{3}$ ceramics synthesized under high pressure, Appl. Phys. Lett. 91 (2007) 172502, https://doi.org/10.1063/ 1.2800816 .

[13] F. Yen, C. dela Cruz, B. Lorenz, E. Galstyan, Y.Y. Sun, M. Gospodinov, C.W. Chu, Magnetic phase diagrams of multiferroic hexagonal $\mathrm{RMnO}_{3}(\mathrm{R}=\mathrm{Er}, \mathrm{Yb}, \mathrm{Tm}$, and Ho), J. Mater. Res. 22 (2007) $2163-2173$, https://doi.org/10.1557/ jmr.2007.0271

[14] N.E. Massa, L. Del Campo, D.D.S. Meneses, P. Echegut, M.J. Martínez-Lope, J. A. Alonso, Phonons and hybrid modes in the high and low temperature far infrared dynamics of hexagonal $\mathrm{TmMnO}_{3}$, J. Phys. Condens. Matter 26 (2014) 275901, https://doi.org/10.1088/0953-8984/26/27/275901

[15] M. Tachibana, T. Shimoyama, H. Kawaji, T. Atake, E. Takayama-Muromachi, JahnTeller distortion and magnetic transitions in perovskite $\mathrm{RMnO}_{3}(\mathrm{R}=\mathrm{Ho}, \mathrm{Er}, \mathrm{Tm}, \mathrm{Yb}$, and Lu), Phys. Rev. B 75 (2007) 2-6, https://doi.org/10.1103/ PhysRevB.75.144425

[16] S. Mukherjee, A. Dönni, T. Nakajima, S. Mitsuda, M. Tachibana, H. Kitazawa, V. Pomjakushin, L. Keller, C. Niedermayer, A. Scaramucci, M. Kenzelmann, E-type noncollinear magnetic ordering in multiferroic o- $\mathrm{LuMnO}_{3}$, Phys. Rev. B 95 (2017) 104412, https://doi.org/10.1103/PhysRevB.95.104412

[17] V.Y. Pomjakushin, M. Kenzelmann, A. Dönni, A.B. Harris, T. Nakajima, S. Mitsuda, M. Tachibana, L. Keller, J. Mesot, H. Kitazawa, E. Takayama-Muromachi, Evidence for large electric polarization from collinear magnetism in $\mathrm{TmMnO}_{3}$, New J. Phys. 11 (2009), https://doi.org/10.1088/1367-2630/11/4/043019

[18] T.C. Han, H.H. Chao, Observation of large electric polarization in orthorhombic $\mathrm{TmMnO}_{3}$ thin films, Appl. Phys. Lett. 97 (2010), https://doi.org/10.1063/ 1.3524500 .

[19] M. Garganourakis, Y. Bodenthin, R.A. De Souza, V. Scagnoli, A. Dönni, M. Tachibana, H. Kitazawa, E. Takayama-Muromachi, U. Staub, Magnetic and electronic orderings in orthorhombic $\mathrm{RMnO}_{3}(\mathrm{R}=\mathrm{Tm}$, Lu) studied by resonant soft x-ray powder diffraction, Phys. Rev. B 86 (2012) 1-5, https://doi.org/10.1103/ PhysRevB.86.054425

[20] Y.W. Windsor, M. Ramakrishnan, L. Rettig, A. Alberca, E.M. Bothschafter, U. Staub, K. Shimamoto, Y. Hu, T. Lippert, C.W. Schneider, Interplay between magnetic order at $\mathrm{Mn}$ and $\mathrm{Tm}$ sites alongside the structural distortion in multiferroic films of o-TmMnO 3, Phys. Rev. B 91 (2015) 235144, https://doi.org/10.1103/ PhysRevB.91.235144

[21] K. Shimamoto, S. Mukherjee, N.S. Bingham, A.K. Suszka, T. Lippert C. Niedermayer, C.W. Schneider, Single-axis-dependent structural and multiferroic properties of orthorhombic $\mathrm{RMnO}_{3}(\mathrm{R}=\mathrm{Gd}-\mathrm{Lu})$, Phys. Rev. B 95 (2017) 1-9, https://doi.org/10.1103/PhysRevB.95.184105

[22] E. Granado, A. García, J.A. Sanjurjo, C. Rettori, I. Torriani, F. Prado, R.D. Sánchez, A. Caneiro, S.B. Oseroff, Magnetic ordering effects in the Raman spectra of $\mathrm{La}_{1-\mathrm{x}} \mathrm{Mn}_{1-\mathrm{x}} \mathrm{O}_{3}$, Phys. Rev. B 60 (1999) 11879-11882, https://doi.org/10.1103/ PhysRevB.60.11879

[23] J. Laverdi ère, S. Jandl, A.A. Mukhin, V.Y. Ivanov, V.G. Ivanov, M.N. Iliev, Spinphonon coupling in orthorhombic RMO3 $(\mathrm{R}=\mathrm{Pr}, \mathrm{Nd}, \mathrm{Sm}, \mathrm{Eu}, \mathrm{Gd}, \mathrm{Tb}, \mathrm{Dy}, \mathrm{Ho}, \mathrm{Y})$ : a Raman study, Phys. Rev. B 73 (2006) 214301, https://doi.org/10.1103/ PhysRevB.73.214301

[24] R. Basistyy, T.N. Stanislavchuk, A.A. Sirenko, A.P. Litvinchuk, M. Kotelyanskii, G. L. Carr, N. Lee, X. Wang, S.W. Cheong, Infrared-active optical phonons and magnetic excitations in the hexagonal manganites $\mathrm{RMnO}_{3}(\mathrm{R}=\mathrm{Ho}, \mathrm{Er}, \mathrm{Tm}, \mathrm{Yb}$, and Lu), Phys. Rev. B 90 (2014) 1-12, https://doi.org/10.1103/PhysRevB.90.024307

[25] F. Sugawara, S. lida, Y. Syono, S. Akimoto, New magnetic perovskites $\mathrm{BiMnO}_{3}$ and $\mathrm{BiCrO}_{3}$, J. Phys. Soc. Japan. 20 (1965) 1529, https://doi.org/10.1143/ JPSJ.20.1529, 1529

[26] F. Sugawara, S. liida, Y. Syono, S. Akimoto, Magnetic properties and crystal distortions of $\mathrm{BiMnO}_{3}$ and $\mathrm{BiCrO}_{3}$, J. Phys. Soc. Japan. 25 (1968) 1553-1558, https://doi.org/10.1143/JPSJ.25.1553

[27] S. Niitaka, M. Azuma, M. Takano, E. Nishibori, M. Takata, M. Sakata, Crystal structure and dielectric and magnetic properties of $\mathrm{BiCrO}_{3}$ as a ferroelectromagnet, Solid State lonics 172 (2004) 557-559, https://doi.org/10.1016/.jssi.2004.01.060

[28] M. Wojdyr, Fityk : a general-purpose peak fitting program, J. Appl. Crystallogr. 43 (2010) 1126-1128, https://doi.org/10.1107/S0021889810030499

[29] K. Yoshii, H. Abe, Magnetic properties of $\mathrm{LnMnO}_{3}(\mathrm{Ln}=\mathrm{Ho}, \mathrm{Er}, \mathrm{Tm}, \mathrm{Yb}$, and $\mathrm{Lu})$, J. Solid State Chem. 165 (2002) 131-135, https://doi.org/10.1006/ jssc. 2001.9514

[30] J.L. Jin, X.Q. Zhang, H. Ge, Z.H. Cheng, Rotating field entropy change in hexagonal $\mathrm{TmMnO}_{3}$ single crystal with anisotropic paramagnetic response, Phys. Rev. B 85 (2012) 1-5, https://doi.org/10.1103/PhysRevB.85.214426

[31] X.M. Wang, Z.Y. Zhao, C. Fan, X.G. Liu, Q.J. Li, F.B. Zhang, L.M. Chen, X. Zhao, X. F. Sun, Low-temperature heat transport, specific heat, and magnetic properties of the hexagonal $\mathrm{TmMnO}_{3}$ single crystals, Phys. Rev. B 86 (2012) 174413, https:// doi.org/10.1103/PhysRevB.86.174413

[32] R.P. Bauman, D.L. Rousseau, S.P.S. Porto, R.P. Bauman, S.P.S. Porto, D. L. Rousseau, S.P.S. Porto, Normal mode determination in crystals, J. Raman Spectrosc. 10 (1981) 253-290, https://doi.org/10.1002/jrs.1250100152

[33] A. De Andrés, J.L. Martínez, J.M. Alonso, E. Herrero, C. Prieto, J.A. Alonso, F. Agulló, M. García-Hern ández, Raman phonons in orthorhombic manganites, 
J. Magn. Magn Mater. 196 (1999) 453-454, https://doi.org/10.1016/S0304-8853 (98)00810-5 .

[34] M.N. Iliev, M.V. Abrashev, J. Laverdi ère, S. Jandl, M.M. Gospodinov, Y.-Q. Wang, Y.-Y. Sun, Distortion-dependent Raman spectra and mode mixing in $\mathrm{RMnO}_{3}$ perovskites ( $\mathrm{R}=\mathrm{La}$, Pr, Nd, Sm, Eu, Gd, Tb, Dy, Ho, Y), Phys. Rev. B 73 (2006), 064302, https://doi.org/10.1103/PhysRevB.73.064302

[35] L. Martín-Carr ón, A. De Andrés, M.J. Martínez-Lope, M.T. Casais, J.A. Alonso, Raman phonons as a probe of disorder, fluctuations, and local structure in doped and undoped orthorhombic and rhombohedral manganites, Phys. Rev. B 66 (2002) 1-8, https://doi.org/10.1103/PhysRevB.66.174303

[36] M. Balkanski, R.F.R. Wallis, E. Haro, Anharmonic effects in light scattering due to optical phonons in silicon, Phys. Rev. B 28 (1983) 1928-1934, https://doi.org/ 10.1103/PhysRevB.28.1928

[37] H.-M. Eiter, P. Jaschke, R. Hackl, A. Bauer, M. Gangl, C. Pfleiderer, Raman study of the temperature and magnetic-field dependence of the electronic and lattice properties of MnSi, Phys. Rev. B 90 (2014), 024411, https://doi.org/10.1103/ PhysRevB.90.024411

[38] S. Mansouri, S. Jandl, A. Mukhin, V.Y. Ivanov, A. Balbashov, A comparative Raman study between $\mathrm{PrMnO}_{3}, \mathrm{NdMnO}_{3}, \mathrm{TbMnO}_{3}$ and DyMnO 3 , Sci. Rep. 7 (2017) 13796, https://doi.org/10.1038/s41598-017-12714-8

[39] J. Vermette, S. Jandl, M.M. Gospodinov, Raman study of spin-phonon coupling in $\mathrm{ErMnO}_{3}$, J. Phys. Condens. Matter 20 (2008) 425219, https://doi.org/10.1088/ 0953-8984/20/42/425219

[40] D.J. Lockwood, M.G. Cottam, The spin-phonon interaction in $\mathrm{FeF}_{2}$ and $\mathrm{MnF}_{2}$ studied by Raman spectroscopy, J. Appl. Phys. 64 (1988) 5876.

[41] W. Baltensperger, J.S. Helman, Influence of magnetic order in insulators on the optical phonon frequency, Helv. Phys. Acta 41 (1968) 668-673, https://doi.org/ $10.5169 /$ seals-113910 Nutzungsbedingungen.
[42] R.X. Silva, H. Reichlova, X. Marti, D.A.B. Barbosa, M.W. Lufaso, B.S. Araujo, A. P. Ayala, C.W.A. Paschoal, Spin-phonon coupling in $\mathrm{Gd}\left(\mathrm{Co}_{1 / 2} \mathrm{Mn}_{1 / 2}\right) \mathrm{O}_{3}$ perovskite, J. Appl. Phys. 114 (2013) 194102, https://doi.org/10.1063/1.4829902

[43] R.B. Macedo Filho, A. Pedro Ayala, C. William de Araujo Paschoal, Spin-phonon coupling in $\mathrm{Y}_{2} \mathrm{NiMnO}_{6}$ double perovskite probed by Raman spectroscopy, Appl. Phys. Lett. 102 (2013) 192902, https://doi.org/10.1063/1.4804988

[44] B.S. Araújo, A.M. Arévalo-López, C.C. Santos, J.P. Attfield, C.W.A. Paschoal, A. P. Ayala, Spin-phonon coupling in monoclinic $\mathrm{BiCrO}_{3}$, J. Appl. Phys. 127 (2020) 114102, https://doi.org/10.1063/1.5143347

[45] E. Aytan, B. Debnath, F. Kargar, Y. Barlas, M.M. Lacerda, J.X. Li, R.K. Lake, J. Shi, A.A. Balandin, Spin-phonon coupling in antiferromagnetic nickel oxide, Appl. Phys. Lett. 111 (2017) 252402, https://doi.org/10.1063/1.5009598

[46] B.S. Araújo, A.M. Arévalo-López, J.P. Attfield, C.W.A. Paschoal, A.P. Ayala, Spinphonon coupling in melanothallite $\mathrm{Cu}_{2} \mathrm{OCl}_{2}$, Appl. Phys. Lett. 113 (2018) 222901 https://doi.org/10.1063/1.5054928

[47] S. Harikrishnan, S. Rößler, C.M. Naveen Kumar, H.L. Bhat, U.K. Rößler, S. Wirth, F. Steglich, S. Elizabeth, Phase transitions and rare-earth magnetism in hexagonal and orthorhombic DyMnO 3 single crystals, J. Phys. Condens. Matter 21 (2009), 096002, https://doi.org/10.1088/0953-8984/21/9/096002

[48] O. Prokhnenko, R. Feyerherm, E. Dudzik, S. Landsgesell, N. Aliouane, L.C. Chapon, D.N. Argyriou, Enhanced ferroelectric polarization by induced by spin order in multiferroic DyMnO 3 , Phys. Rev. Lett. 98 (2007), 057206, https://doi.org/ 10.1103/PhysRevLett.98.057206

[49] J. Xu, J.H. Park, H.M. Jang, Orbital-spin-phonon coupling in jahn-teller-distorted $\mathrm{LaMnO}_{3}$ : softening of the 490 and $610 \mathrm{~cm}^{-1}$ Raman-active modes, Phys. Rev. B 75 (2007) 10-13, https://doi.org/10.1103/PhysRevB.75.012409 